\title{
Differential diagnosis and management of oral ulcers
}

\author{
Amanda Siu, DDS, ${ }^{1}$ Katina Landon, DDS, ${ }^{2}$ and Daniel M Ramos, DDS, PhD ${ }^{2}$
}

\section{Abstract}

The diagnosis and treatment of oral lesions is often challenging due to the clinician's limited exposure to the conditions that may cause the lesions and their similar appearances. While many oral ulcers are the result of chronic trauma, some may indicate an underlying systemic condition such as a gastrointestinal dysfunction, malignancy, immunologic abnormality, or cutaneous disease. Correctly establishing a definitive diagnosis is of major importance to clinicians who manage patients with oral mucosal disease. Some of these diseases are infectious; however, most are chronic, symptomatic, and desquamative. Treatment and management requires an understanding of the immunopathologic nature of the lesion. This review will address how to differentiate and diagnose varying types of oral ulcers and provide a treatment strategy.

Semin Cutan Med Surg 34:171-177 @ 2015 Frontline Medical Communications

W hile many oral ulcers are the result of chronic trauma, some may indicate an underlying systemic condition such as a gastrointestinal dysfunction, malignancy, immunologic abnormality, or cutaneous disease. ${ }^{1}$ Herein, we will discuss how to differentiate and diagnose varying types of oral ulcers and provide treatment strategies.

\section{Neoplasm}

\section{Oral squamous cell carcinoma}

Oral squamous cell carcinoma (SCC) presents in several clinical forms: either as a white lesion (leukoplakia), a red lesion (erythroplakia), a red and white lesion (erythroleukoplakia), an indurated mass, or as a mucosal ulcer. The floor of the mouth (Figure 1A) and lateral tongue (Figure 1B) are the most common intraoral sites for this malignancy. The major risk factors for oral cancer include tobacco use and alcohol consumption. SCC of the lip, associated with ultraviolet (UV) light and pipe smoking, often presents as a chronic, nonhealing ulcer (Figure 1C) at the vermillion border. This form of cancer can be similar in appearance to several other ulcerative conditions, including recurrent herpes simplex virus 1 (HSV-1). ${ }^{2}$ Cancer of the lower lip has a 5-year survival rate of $90 \%$, which is higher than the generally quoted 5-year survival rate for intraoral cancer of $40 \%$ to $50 \% .^{2}$ Approximately $80 \%$ of tongue

${ }^{1}$ Department of Pediatric Dentistry, University of Texas, Health Science Center, San Antonio.

${ }^{2}$ Department of Orofacial Sciences, University of California at San Francisco. Disclosures: The authors have nothing to disclose.

Correspondence: Daniel M Ramos, DDS, PhD; Department of Orofacial Sciences; University of California; 513 Parnassus Ave - S612; Box 0422; San Francisco, CA 94143-0422. Email: Daniel.ramos@ ucsf.edu.
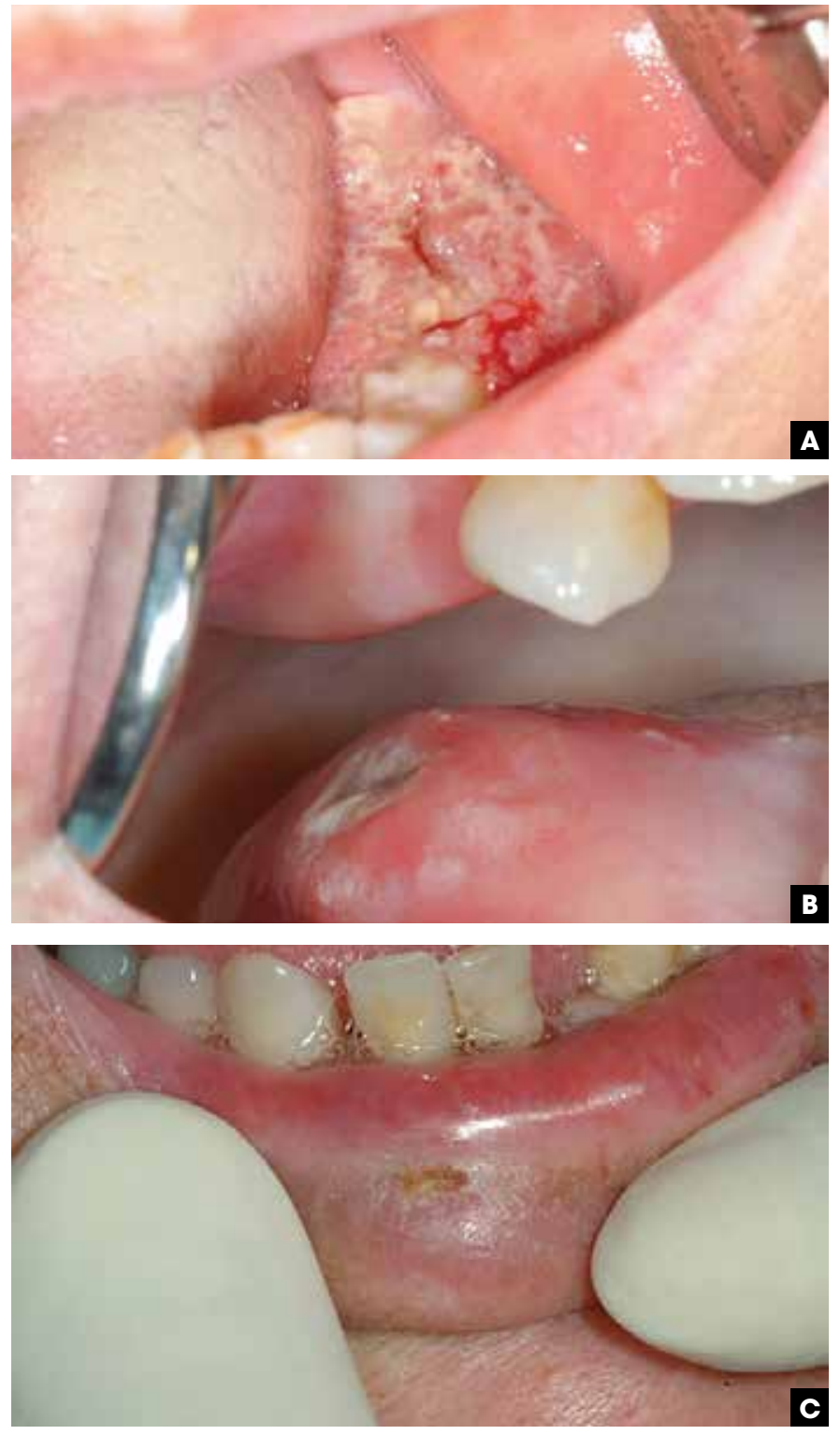

FIGURE 1. Squamous Cell Carcinoma (SCC).A) SCC on the floor of the mouth. Patient had significant pain on the left floor of the mouth. The floor was ulcerated and comprised of mixed hyperplastic/hyperkeratotic tissue. B) SCC of the lateral border of the tongue. Ulcerated mass on the right posterior-lateral border of tongue was biopsied and proven to be SCC. Patient history significant for poorly controlled erosive lichen planus. C) Squamous cell carcinoma of lower lip. An asymptomatic, non-healing ulcer was present for 4 months on the lower lip. Patient had a history of unprotected outdoor activity. Biopsy proved the lesion to be squamous cell carcinoma. 


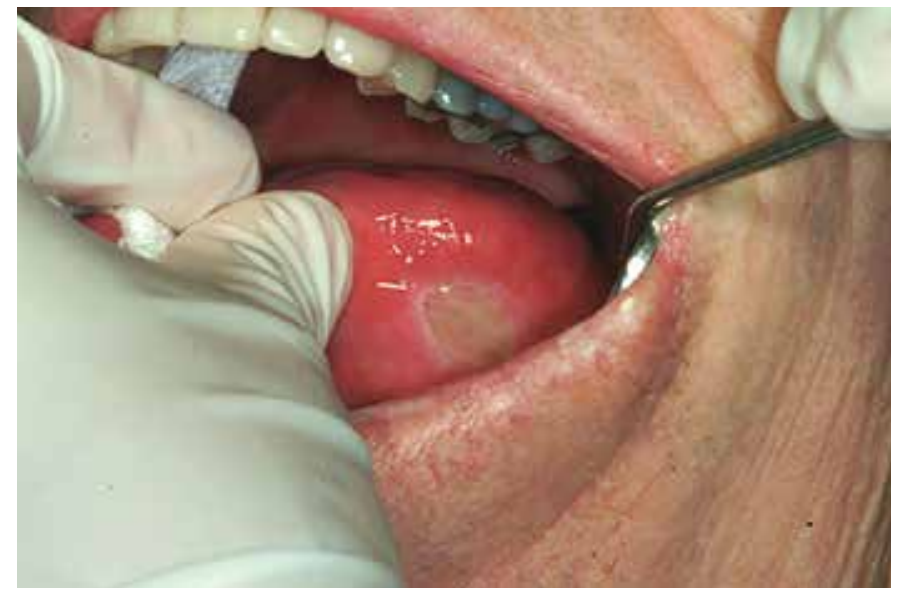

FIGURE 2. Traumatic ulcer on the tongue. Dental filling material was inadvertently left in between the lower left teeth, which resulted in chronic trauma and the formation of a large latrogenic ulcer on the tongue. Patient has paresthesia on the left side and could not feel the ulcer forming.

cancers have nodal metastases at time of diagnosis contributing to the poorer 5 -year survival rate. ${ }^{2}$ SCC of the oral cavity can mimic a variety of benign conditions occurring at multiple sites. Therefore, a careful soft tissue examination should be performed at each dental or medical appointment. Any ulcer that is present longer than 2 weeks and cannot be explained should be further evaluated and biopsied.

\section{Reactive lesions}

Trauma is the most common cause of oral ulcers. Traumatic ulcers may result from either exposure to habits, chemicals, heat, or repeated mechanical friction. They may occur at any age and in either sex. ${ }^{3}$ Traumatic ulcers may also result after dental treatment from mucosal injury while the patient is still anesthetized. Mucosal injury can occur by contact with a piece of sharp dental composite material (Figure 2). Self-induced injuries (factitial) are rare and notoriously difficult to diagnose. These injuries can be caused by a parafunctional habit such as picking the attached gingiva with

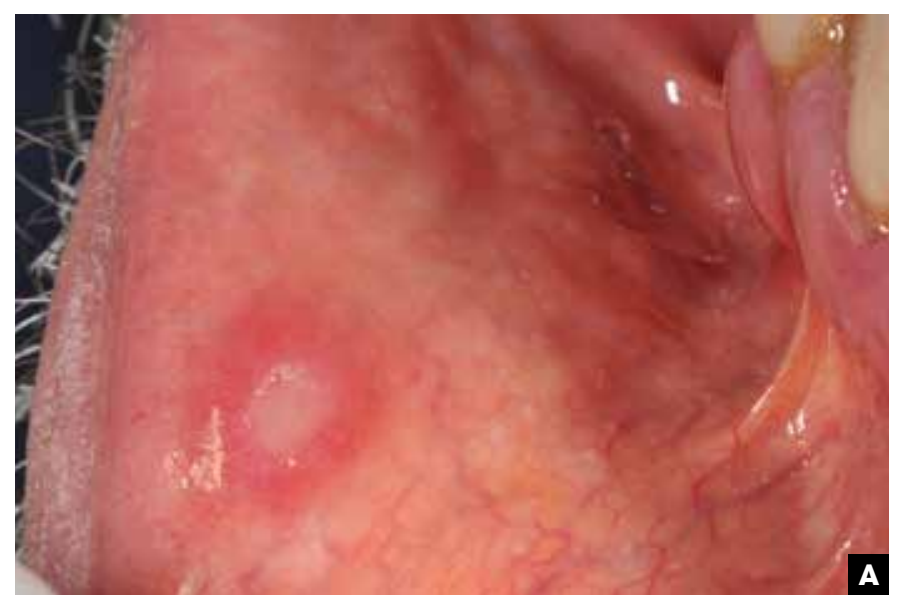

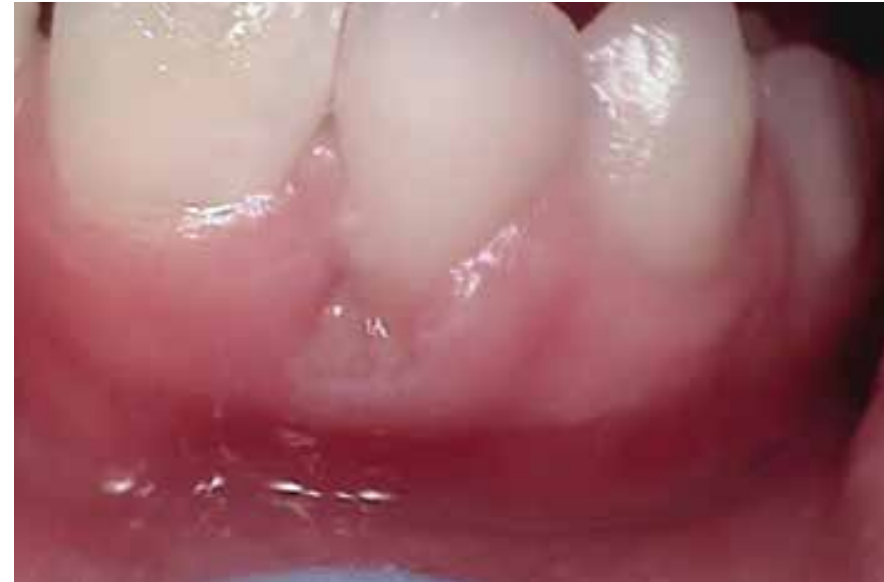

FIGURE 3. Factitial injury of lower gingiva. Repeated digging at the gingival collar as an unconscious habit resulting in localized trauma, gingival recession, and ulcer formation.

a fingernail (Figure 3). A traumatic ulcer should resolve within 2 weeks after removing the source of the injury; but any unexplained ulcer that does not resolve in 2 weeks, must be biopsied for microscopic analysis.

\section{Ulcers with white or red changes: recurrent aphthous stomatitis}

Recurrent aphthous ulcers (RAS), colloquially referred to as 'canker sores,' can be classified into 3 categories according to their size and presentation. Minor aphthae may be single or multiple, ranging in size between $2-5 \mathrm{~mm}$, are shallow, and do not scar on healing (Figure 4A). In contrast, major aphthae are typically solitary, greater than $5 \mathrm{~mm}$ in diameter, deep, and can scar upon healing (Figure 4B). Minor and major aphthae occur on nonkeratinized tissues such as the labial and buccal mucosae, alveolar mucosa, and soft palate. Herpetiform aphthous ulcers are an unusually small variant $(<1 \mathrm{~mm})$ that tend to occur in clusters. All forms of aphthous ulcers have a yellow-gray pseudomembrane covering, rounded shape, and a red (erythematous) halo. The pathogenesis of aphthous ulcers is not well defined, but alterations in local cell-

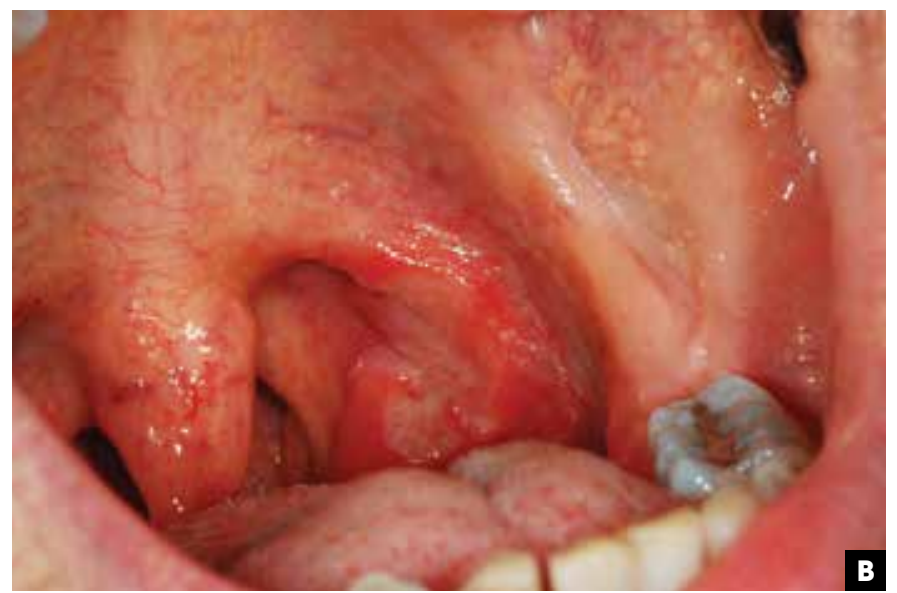

FIGURE 4. Aphthous stomatitis. A) Example of a classic minor aphthous ulcer appearing on the lower labial mucosa. The lesion is well defined with a psuedomembrane covering, with an erythematous halo surrounding the ulcer. The typical size is $<5 \mathrm{~mm}$. B) Example of a major aphthous ulcer on the left tonsillar fossae. Lesion is irregular in shape and deep. Scarring can occur. The typical size is $>5 \mathrm{~mm}$. 

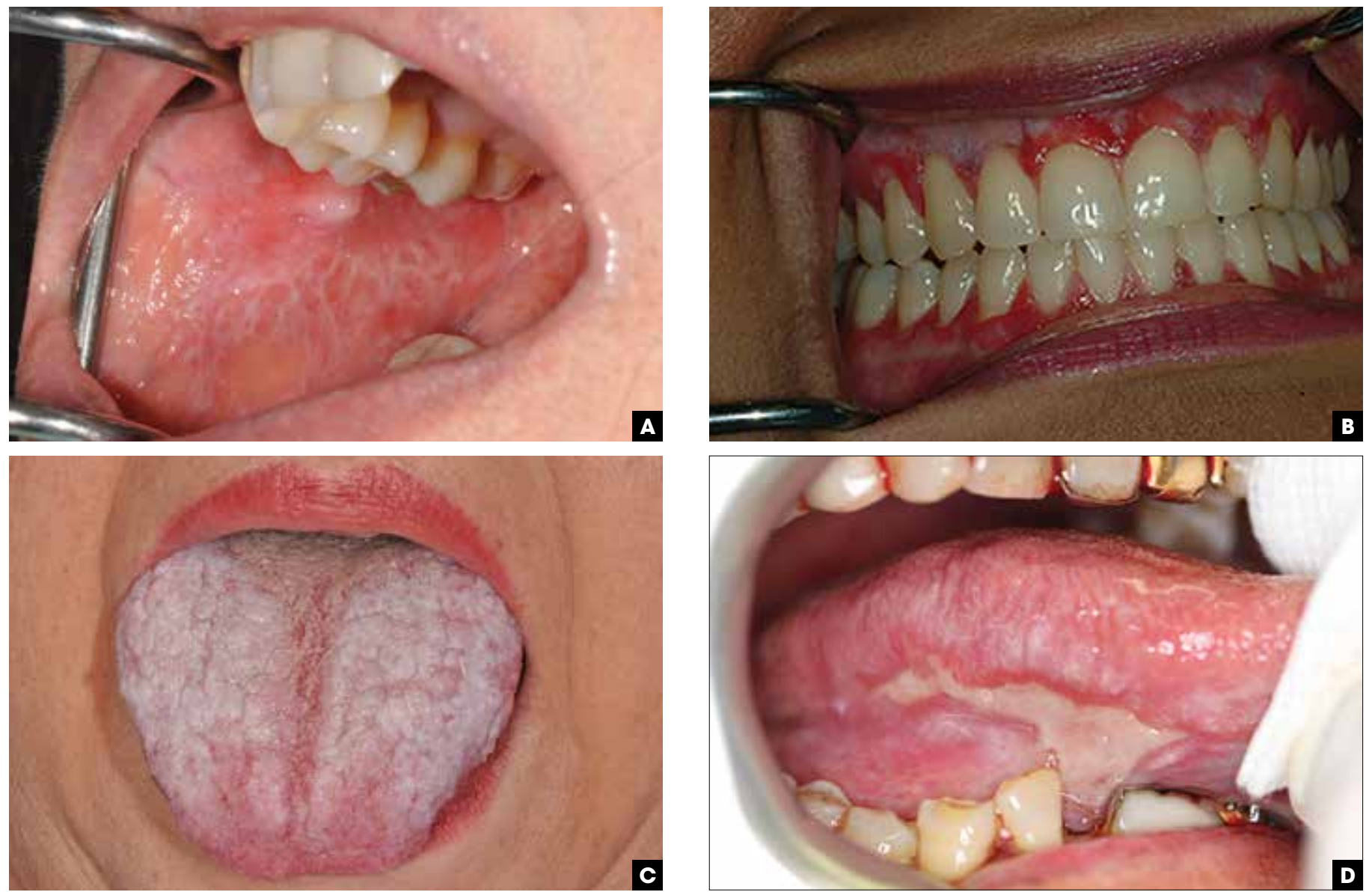

FIGURE 5. Lichen planus. A) Reticular form: Example of Wickham's Striae on the buccal mucosa of a lichen planus patient. Notice the lattice-like appearance of the most common form. B) Atrophic form: Example of atrophic form of lichen planus that can occur exclusively on the gingiva. Notice the widespread appearance that appears to be localized to the attached gingiva. C) Plaque form: Example of plaque-form of lichen planus on the tongue of patient which is similar in appearance to keratosis. It may be more prevalent in smokers. D) Erosive form: Example of a large map-like lesion of oral lichen planus on the ventral surface of the tongue. Notice hyperkeratosis surrounding ulcer.

mediated immunity may be important. ${ }^{4}$ Since both minor and herpetiform aphthous ulcers typically resolve in 7-10 days, treatment is varied and palliative. Treatments have included tetracycline or doxycycline mouth rinses, topical corticosteroids (fluocinonide), and silver nitrate cauterization. The latter reduces the pain of aphthae via necrosis of small nerve fibers, but usually results in prolonged healing. Intralesional or oral corticosteroids are indicated for major aphthae. For treatment of widespread, persistent disease; oral corticosteroids may be coupled with steroid-sparing drugs such as azathioprine or mycophenylate. Colchicine, dapsone, and pentoxifylline have also been used for major aphthae with varying degrees of effectiveness. ${ }^{5}$

\section{Systemic disorders associated with recurrent aphthous stomatitis}

Several systemic problems can present with oral aphthous ulcers including: Behçet's syndrome, celiac disease, cyclic neutropenia, nutritional deficiencies, Immunoglobulin A (IgA) deficiency, MAGIC syndrome (mouth and genital ulcers with inflamed cartilage), and Sweet syndrome (febrile neutrophilic dermatosis). In these conditions, aphthous ulcers appear clinically similar to those in patients without concurrent systemic diseases. It is important to ask patients about any diagnosed or undiagnosed gastrointestinal issues. If oral ulcers become chronic, they may represent a more serious problem.

\section{Oral lichen planus}

Oral lichen planus (LP) is a mucosal presentation of lichen planus that has a variety of clinical features. Oral LP can present as reticular (white papules and plaques; Figure 5A), atrophic (erythematous; Figure 5B), plaque-like (Figure 5C), and/or erosive oral LP (erosions and ulcers; Figure 5D). Reticular LP is usually asymptomatic. Pain is more often associated with the erythematous and erosive forms of LP. The most common intraoral site is the buccal mucosa; however, the tongue, lips, palate, gingiva, and floor of the mouth can also be affected. Microscopically, LP is characterized by hyperkeratosis, degeneration of the basal cell layer of the epithelium, and the presence of a subepithelial band of lymphocytes (interface mucositis). The reliable demonstration of fibrinogen depositions beneath the basement membrane by direct immunofluorescence (DIF) is a useful diagnostic test. Patients with signs or symptoms suggesting involvement of other mucosal sites should be referred to an appropriate specialist for an evaluation. For asymptomatic cases, no treatment is needed; but for those patients 


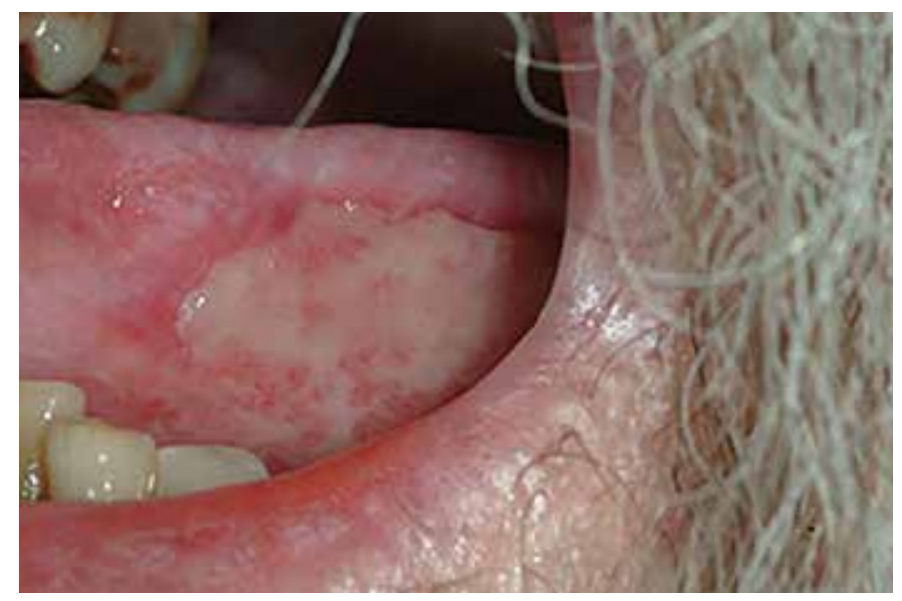

FIGURE 6. Pemphigus Ulceration: Example of a large psuedomembrane covered ulcer on the lateral border of the tongue due to pemphigus vulgaris.

with symptoms and/or the presence of ulceration, either topical or systemic corticosteroids may be used as treatment options.

\section{Vesiculo-bullous lesions \\ Pemphigus vulgaris}

Pemphigus vulgaris (PV) belongs to a family of disorders that form bullae on the skin or mucous membrane. PV is an acantholytic disorder caused by circulating antibodies against the desmosome protein, desmoglein-3, resulting in the formation of intraepithelial blisters. Four major PV types are recognized: PV, pemphigus foliaceus, IgA pemphigus, and paraneoplastic pemphigus. PV is the most common form seen intraorally. ${ }^{6} \mathrm{PV}$ usually occurs in the $4^{\text {th }}$ or $5^{\text {th }}$ decade of life with oral lesions preceding the onset of cutaneous lesions. When bullae rupture, the resultant ulcers are often large and may coalesce (Figure 6). ${ }^{7}$ Lesions can range from the size of a small aphthous ulcer $(<5 \mathrm{~mm})$ to a large, painful, psuedomembrane-covered lesion $(2 \mathrm{~cm})$. The severity of oral lesions can prevent adequate nutrition due to severe pain during food consumption. Clinically, PV must be distinguished from other erosive mucosal diseases such as, mucous membrane pem- phigoid, erosive LP, and erythema multiforme. Diagnosis requires a biopsy to identify the location of the epithelial separation (acantholysis), and usually DIF to localize the autoantibody attached to the tissue in the suprabasal areas of the stratum spinosum (IgG and C3). ${ }^{8}$ Almost all cases of $\mathrm{PV}$ require systemic corticosteroids often supplemented with nonsteroidal immunosuppressants such as, azathioprine or mycophenolate. For less severe cases with limited oral involvement, topical corticosteroids may be used; but this is uncommon.

\section{Mucous membrane pemphigoid}

Far more common than PV, mucous membrane pemphigoid (MMP) is a chronic blistering disease that affects the oral and ocular mucous membranes. In the oral cavity, the gingiva and buccal mucosa are the most commonly affected sites. Mucous membrane pemphigoid typically occurs in the $5^{\text {th }}$ and $6^{\text {th }}$ decade of life. In contrast to PV, where the blisters are fragile, intact bullae are more commonly seen in MMP (Figure 7A). Lesions can be isolated to the gingiva and sometimes referred to as desquamative or erosive gingivitis (Figure 7B). In some mild cases of MMP, only gingival erythema and edema is observed and mistaken by dentists as a variety of gingivitis. The diagnosis of MMP and long term care typically includes an ophthalmologist in the treatment team since eye lesions are frequent and scarring of the canthus (symblepharon), corneal scarring, and eyelash inversion (entropion) can lead to visual complications. Microscopically, epithelial separation at the level of the basement membrane without acantholysis is seen. DIF shows a linear distribution of IgG and C3 localized at the basement membrane to the target proteins laminin-5 and bullous pemphigoid antigen-180 (BP-180). Therapy for MMP includes the use of topical or systemic corticosteroids depending on the severity and extent of the disease. In some refractory instances, corticosteroid treatment is coupled with nonsteroidal immunosuppressants such as azathioprine or mycophenolate.

\section{Erythema multiforme}

Erythema multiforme (EM) is an acute, hypersensitivity reaction characterized by the appearance of distinctive target-like lesions on the skin that can be accompanied by erosions of the oral and/
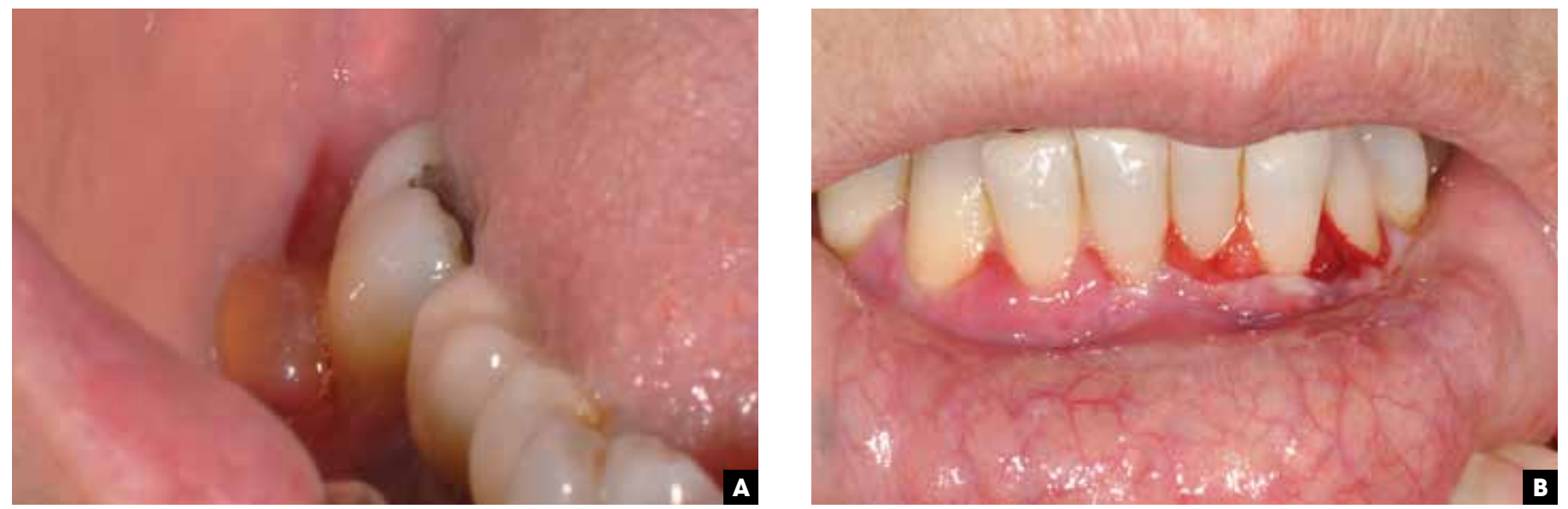

FIGURE 7. Pemphigus vulgaris. A) Bulla: Example of short-lived bullae on the gingival tissue area buccal to tooth \#31. B) Mucous membrane pemphigoid. Gingival ulceration: Example of gingival ulceration and sloughing due to an uncontrolled mucous membrane pemphigoid. Note the generalized gingival recession due to the disease. Plaque control is essential to reduce the impact of the disease. 

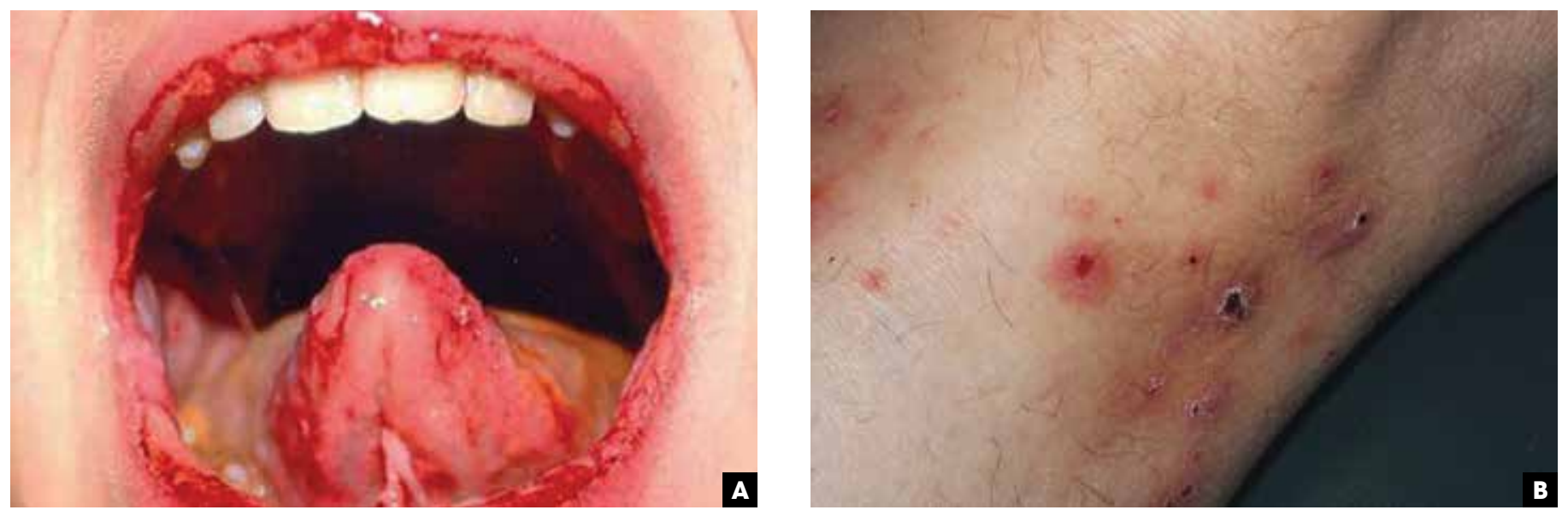

FIGURE 8. Erythema multiforme (EM). A) Oral EM: Example of the widespread ulcerations seen in EM. Ulcerations can be found on any surface including the lips, tongue, and buccal mucosa.

B) Cutaneous EM: Example of typical "target" skin lesions associated with EM.

or genital mucosa. Erythema multiforme major, a response to a drug exposure, has both cutaneous and mucous membrane involvement. Erythema multiforme minor, a response to HSV infection, has no mucosal presentation. Intraoral lesions can present as large pseudomembrane-covered ulcers on the buccal mucosa, ventral tongue, and labial vestibule (Figure 8A). Hemorrhagic crusting of the vermillion border of the lips is characteristic of EM. Cutaneous lesions (Figure 8B) usually accompany those found in the oral cavity lesions although the oral mucosa can be solely affected. EM typically affects young adults with a slight predilection for males. The precise mechanism of EM is not completely understood although it most likely involves the formation of antigen-antibody complexes. ${ }^{9,10}$ Skin lesions, when present, can have a variety of presentations (multiforme) including the classic target lesions or macule-type lesions. Treatment of both minor and major EM consists of corticosteroids and sometimes acyclovir. It is now thought that Stevens-Johnson syndrome (SJS) and toxic epidermal necrolysis (TEN) are more severe manifestations of bullous EM. SJS has been defined as skin detachment of $<10 \%$ of the body surface; whereas $>30 \%$ skin detachment is found in TEN. Patients with SJS/TEN are at high risk of sepsis and septic shock ( $S$. aureus and $P$. aeruginosa), which are the main cause of death in these patients. Due to the high risk of bacterial superinfection and sepsis, appropriate cultures should be performed from blood, wounds, and mucosal lesions. In children, Mycoplasma pneumoniae infection should also be determined.

\section{Viral causes of ulceration \\ Herpes simplex virus 1}

Primary herpetic gingivostomatitis is the most common viral cause of oral ulcers. Affected individuals typically are febrile with widespread superficial ulcers of the oral mucosa both keratinized and non-keratinized (Figure 9). The gingiva is often swollen and ulcerated, which give it the features of acute necrotizing ulcerative gingivitis. HSV-1 infection can occur at any age although it is primarily seen during childhood. Therapy is typically palliative, although systemic acyclovir and other antiviral medication should be considered when the disease is severe or recurrent. Approximately $30 \%$ of patients who have primary HSV-1 infection develop recurrent episodes of herpes labialis (cold sore).

\section{Herpes simplex virus 2}

HSV-2 can give rise to oral ulcers similar to those of primary HSV1 infections and is a consequence of orogenital transmission.

\section{Epstein-Barr virus}

In an immunocompetent individual, oral Epstein-Barr virus (EBV) infections are rare. However, oral EBV infections are more common in the setting of immunosuppression. EBV infections most commonly manifest as the white lesion on the lateral border of the tongue, termed hairy leukoplakia (discussed in White Lesions in the Oral Cavity: Clinical Presentation, Diagnosis and Treatment [this issue]). An unusual form of EBV infection is seen in immunosuppressed individuals termed EBV-positive mucocutaneous ulcer. This is a self-limited, indolent disorder, generally responding well to conservative management. Lesions are histologically characterized by a polymorphous infiltrate and atypical large B-cell blasts often with Hodgkin/Reed-Sternberg (HRS) cell-like morphology. The B cells show strong CD30 and EBER (Epstein-Barr virus encoded small RNA) positivity, some with reduced CD20 expression, in a background of abundant $\mathrm{T}$ cells.

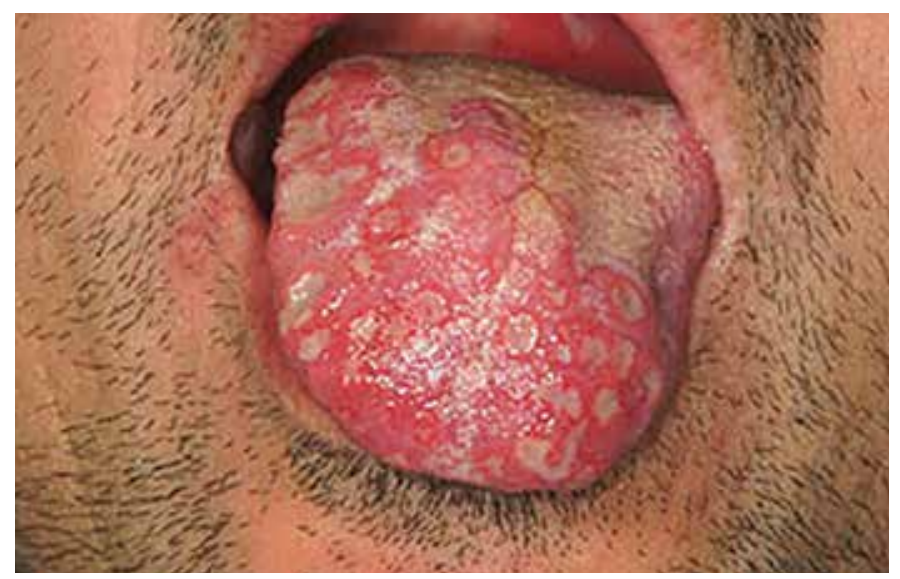

FIGURE 9. Herpes Simplex Virus I. Primary herpetic gingivostomatitis: Example of a primary herpetic outbreak in 35 year old male accompanied by headache, fever and overall malaise. Notice the ruptured vesicles on the tongue and the aphthous-like appearing lesion on the soft palate. 


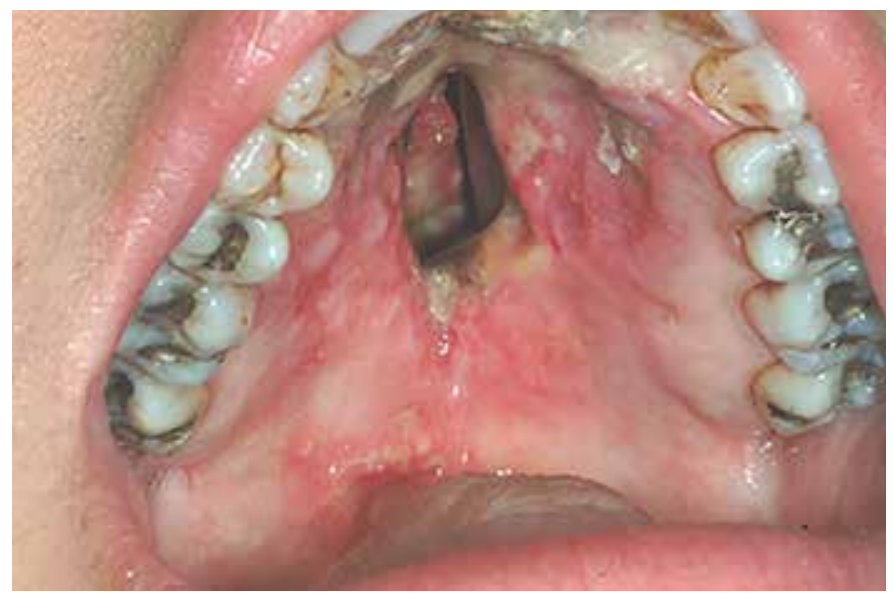

FIGURE 10. Granulomatosis with polyangitis (Wegner's granulomatosis). Example of a palatal perforation in granulomatosis with polyangitis. Note complete erosion of the boney palate.

\section{Human immunodeficiency virus}

Human immunodeficiency virus (HIV) infection can cause a variety of oral ulcers including severe necrotic ulcers of unknown etiology. These ulcers are painful and can cause dysphagia. Buccal and pharyngeal mucosa is most commonly affected. The etiology of these HIV-related ulcers is unknown although initially it was suspected to be due to CMV. Treatments for these ulcers are antiinflammatory drugs like thalidomide and or tumor necrosis factoralpha (TNF囚) blocker.

\section{Bacterial causes of ulceration: Treponema pallidum}

Syphilis is a well-known disorder with a long history in medicine. The disease is caused by the spirochete Treponema pallidum and has 3 temporally distinct phases. Primary syphilis occurs as a chancre at the site of initial infection. Oral chancres are well known as the result of orogenital contact. Chancres initially appear as a small papule that elevates, enlarges, erodes, and becomes ulcerated. The lesion is usually punched-out, indurated and approximately $2-3 \mathrm{~cm}$ in diameter without a red inflammatory border. The surface is covered by a yellowish, highly infectious, serous discharge. Chancres typically last 2 to 4 weeks and heal spontaneously. Secondary syphilis presents with either oral lesions including red macules, pharyngitis, or isolated/multiple painless, shallow, and highly infectious ulcers surrounded by an erythematous halo. The borders are irregular and may resemble "snail tracks." Tertiary syphilis occurs in approximately $30 \%$ of untreated syphilis cases appearing many years after initial infection. It is primarily characterized by gummas, palatal perforation, and neurological symptoms. Penicillin-G remains the drug of choice for treating all stages of syphilis.

\section{Oral ulcers due to systemic conditions Granulomatosis with polyangitis}

Granulomatosis with polyangitis (GPA; Wegner's Granulomatosis) presents as oral ulcers associated with upper respiratory tract, lung, and kidney involvement. ${ }^{11}$ Initial disease presentation typically involves painful cobblestone changes to the mucosal surface of the palate and gingiva (strawberry gingiva). GPA is a form of necrotiz-

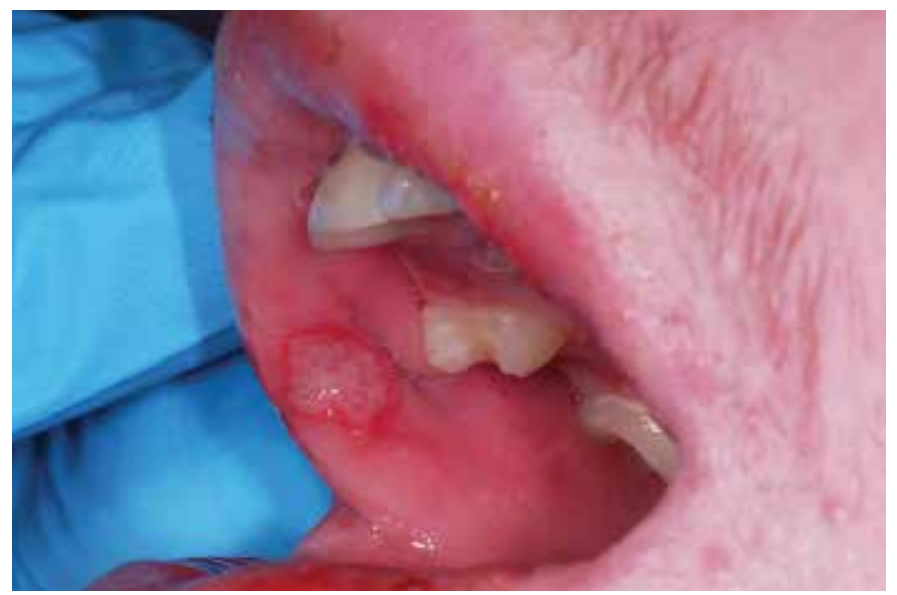

FIGURE 11. Behçet's Syndrome: Example of an aphthous-like ulcer in a patient with Behçet's syndrome.

ing vasculitis with granulomatous inflammation. Advanced GPA can result in palatal perforation (Figure 10). ${ }^{12}$ Cytological examination revealing anti-neutrophil cytoplasmic and peri-nuclear antibodies (cANCA, pANCA) further confirms the diagnosis; but their absence does not necessarily rule out the diagnosis. Treatment includes corticosteroids plus cyclophosphamide.

\section{Behçet's syndrome}

Behçet's disease is a multisystem chronic inflammatory disease of unknown cause. Oral lesions generally appear as diffuse aphthousappearing mucosal erosions (Figure 11). In 2006, the International Criteria for Behçet's Disease (ICBD) established criteria in an attempt to better define the disease based on a point system. Three or more points is used to give a diagnosis of Behçet's Disease (genital aphthosis 2 points, eye lesions 2 points, and the remaining receive one point each [skin, oral aphthous, vascular lesion] $)^{13}$. No consistent treatment for Behçet's syndrome exists. Corticosteroids, azathioprine, thalidomide, and Dapsone have all been used with some success. Success is dependent upon the stage of the disease.

\section{Conclusion}

Diagnosis of oral ulceration can be challenging and requires careful clinical examination and history taking. It is important to understand that oral manifestations may represent part of a larger problem. Biopsy may be indicated to ascertain a true diagnosis. Any unexplained ulcer found in the oral cavity that does not resolve in 2 weeks should be evaluated microscopically.

\section{References}

1. Porter SR, Leao JC, Review article: oral ulcers and its relevance to systemic disorders, Aliment Pharmacol Ther. 2005;21(4):295-306.

2. Regezi JA, Sciubba JJ, Jordan RCK. Oral Pathology: Clinical Pathologic Correlations. $6^{\text {th }}$ ed. St. Louis, Missouri: Saunders/Elsevier; 2012.

3. Woo SB. Oral Pathology: A Comprehensive Atlas and Text. Philadelphia, PA: Elsevier/Saunders; 2012.

4. Langlais RP, Miller CS, Nield-Gehrig JS. Color Atlas of Common Oral Diseases. $4^{\text {th }}$ ed. Walters Kluwer/Lippincott Williams \& Wilkins; 2009.

5. Altenburg A, Abdel-Naser MB, Seeber H, Abdallah M, Zouboulis CC. Practical aspects of management of recurrent aphthous stomatitis. J Eur Acad Dermatol Venereol. 2007;21(8):1019-1026.

6. Mihai S, Sitaru C. Immunopathology and molecular diagnosis of autoimmune bullous diseases. J Cell Mol Med. 2007;11(3):462-481.

7. Mays JW, Sarmadi M, Moutsopoulos NM. Oral manifestations of systemic autoim- 
mune and inflammatory diseases: diagnosis and clinical management. $J$ Evid Based Dent Pract. 2012;(3 Suppl):265-282.

8. Anhalt GJ, Kim SC, Stanlet JR, et al. Paraneoplastic pemphigus. An autoimmune mucocutaneous disease associated with neoplasia. N Engl J Med. 1990;323(25):1729-1735.

9. Farthing P, Bagan JV, Scully C. Mucosal disease series. Number IV. Erythema multiforme, Oral Dis. 2005;11(5):261-267.

10. Scully C, Bagan J. Oral mucosal diseases: erythema multiforme, Br J Maxillofac Surg. 2008;46(2):90-95.

11. Eufinger H, Machtens E, Akuamoa-Boateng E. Oral manifestations of Wegener's granulomatosis. Review of the literature and report of a case. Int J Oral Maxillofac Surg. 1992;21(1):50-53.

12. Silverman S, Eversole LR, Truelove EL. Essentials of Oral Medicine. Hamilton, Ontario: BC Decker, 2001.

13. Davatchi F. Diagnosis/Classification Criteria for Behcet's Disease. Patholog Res Int 2012:607921.
14. Yuan A, Woo SB. Adverse drug events in the oral cavity. Oral Surg Oral Med Oral Pathol Oral Radiol. 2015;119(1):35-47.

15. Scully C, Felix DH, Oral medicine-update for the dental practitioner. Apthous and other common ulcers. Br Dent J. 2005;199(5):259-264.

16. Leão JC, Gomes VB, Porter S. Ulcerative lesions of the mouth: an update for the general medical practitioner. Clinics (Sao Paulo). 2007;62(6):769-780.

17. Bouquot JE, Muller S, Nikai H. Lesions of the oral cavity. In: Diagnostic Surgical Pathology of the Head and Neck. 2nd ed. Philadelphia, PA: Saunders; 2009:111-190.

18. Scully C, Lo Muzio L. Oral mucosal diseases: mucous membrane pemphigoid. $\mathrm{Br} \mathrm{J}$ Oral Maxillofac Surg. 2008;46(5):358-366.

19. Carrozzo M, Togliatto M, Gandolfo S. Erythema multiforme. A heterogeneous pathologic phenotype [in Italian]. Minerva Stomatol. 1999:48(7):217-226.

20. Bruce AJ, Rogers RS. Acute oral ulcers. Dermatol Clin. 2003:21(1):1-15.

21. Scully C, Felix DH. Oral medicine - update for the dental practitioner. Mouth ulcers of more serious connotation. Br Dent J. 2005;199(6):339-343. 